CLINICAL CASE

\title{
ANTERIOR CRUCIATE LIGAMENT RECONSTRUCTION IN POSTERIOR CRUCIATE LIGAMENT DEFICIENT KNEES - WHAT CAN BE SAVED?
}

\author{
T.C. Munteanu ${ }^{1}$, Ana-Raluca Mihalcea ${ }^{1}$, B. Nistor ${ }^{2}$, G. Dinu ${ }^{3}$ \\ 1"Carol Davila" University of Medicine and Pharmacy \\ ${ }^{2}$ Monza Hospital, Bucharest \\ ${ }^{3}$ Orthopedics and Traumatology Clinic, The Emergency Clinical Hospital, Bucharest, Romania \\ Corresponding author: Tiberiu-Ciprian Munteanu \\ Phone no. 0040748207070 \\ E-mail: tiberiumunteanu@yahoo.com
}

\begin{abstract}
The floppy Anterior Cruciate Ligament (ACL) sign is a well-established arthroscopic and MRI indicator of Posterior Cruciate Ligament (PCL) insufficiency. We explored the diagnostic and therapeutic options in such cases by retrospectively analyzing the management of a series of five consecutive cases of iatrogenic floppy ACL reconstructions which resulted in fixed posterior drawer. All patients were young athletes. Because their ACL rehabilitation program was failing they sought for a new medical expertise. In all cases arthroscopic PCL reconstruction was performed and the acquired injuries after the initial ACL reconstruction were treated. All patients regained full range of motion and stable knees. Confusion between a PCL and an ACL injury still seems to be high. If not addressed accordingly, the fixed posterior drawer may lead to irreversible damage of the knee joint.
\end{abstract}

Keywords: anterior cruciate ligament, posterior cruciate ligament, fixed posterior drawer, arthroscopic reconstruction

\section{Introduction}

The floppy Anterior Cruciate Ligament (ACL) sign is a well-established arthroscopic and MRI indicator of Posterior Cruciate Ligament (PCL) insufficiency.

The ACL is attached distally to the front of the intercondyloid eminence of the tibia and proximally to the medial aspect of the lateral femoral condyle (Figure 1) [3]. It is considered that the ACL consists of 2 different bundles, with separate the tibial insertion and function: the anteromedial bundle and the posterolateral bundle [1]. Overall, it acts as the primary restraint against anterior tibial translation on the femur, each bundle being responsible for restricting anterior tibial translation for a precise range of flexion [8]. Also, ACL contributes to limiting hyperextension, medial and lateral rotation and varus/valgus movements of the knee joint [5].

The PCL extends from its proximal insertion on the lateral aspect of the medial femoral condyle down to its posterior area on the tibial plateau, between the posterior horns of the menisci (Figure 1) [8]. Because the distal insertion and the cross-section of the PCL is larger than the ACL, the PCL is able to bear bigger loads than the ACL [7]. The PCL consists also of 2 separate bundles: a larger 
anterolateral and smaller posteromedial [6]. The main function of the PCL is to restrain the posterior translation of the tibia (PTT) on the femur [14]. In full extension, the posteromedial bundle is tight, whereas in 90 degrees of flexion the anterolateral bundle is fully extended. Beyond 90 degrees, the posterolateral bundle increases the force that restricts posterior tibial translation [15]. PTT is maximum between 75 to 90 degrees of flexion. 8 From here, the other structures that limit PTT become ineffective, and PTT depends on the PCL competence. PCL is also involved in varus/valgus and rotational stability.



Figure 1 - Cruciate ligaments viewed from the anterolateral portal

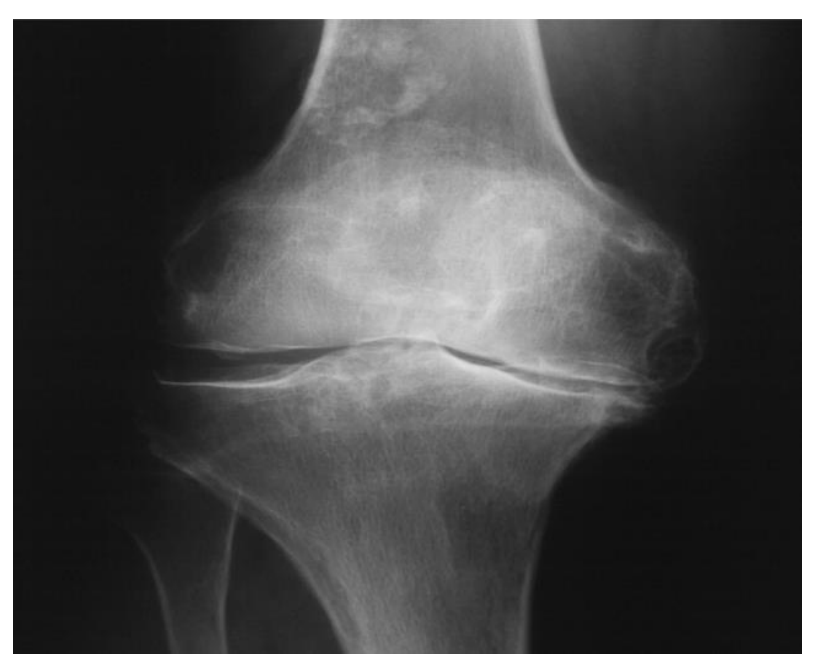

Figure 2 - Coronal anteroposterior view Gonarthrosis

When the PCL is incompetent, the tibia sags posteriorly and the ACL becomes lax. Often, this ACL laxity is mistaken for an ACL injury and ACL reconstruction follows, in the context of a PCL deficient knee. The result is an aggravated PCL insufficiency, with the tibia fixed in a posterior position, due to the ACL graft tensioning [9].

The natural evolution of an unaddressed PCL injury threatens to compromise the knee. Incorrect tibial loading leads eventually to the destruction of the articular cartilage and other joint structures(Figure 2, Figure 3) [16,17].

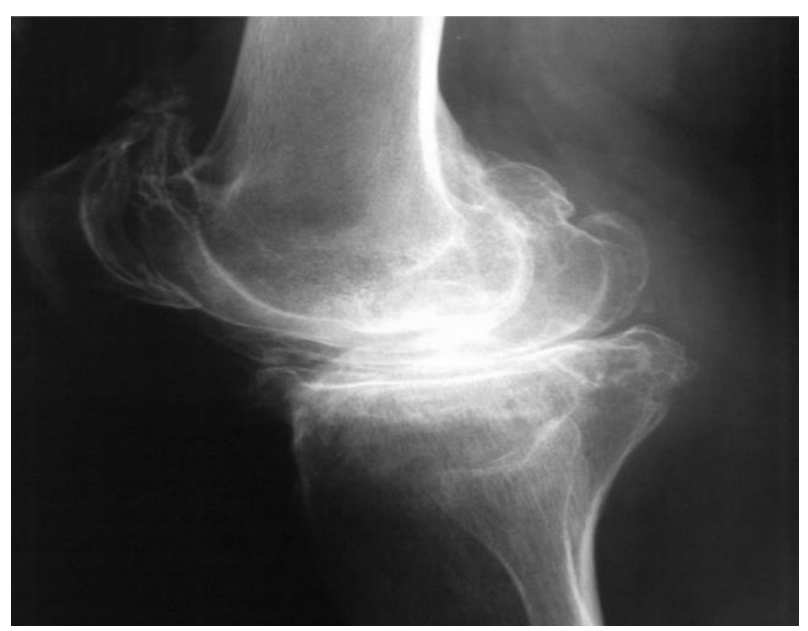

Figure 3 - Sagittal view: femorotibial and patellofemoral - arthrosis

According to Strobel, PCL injuries may be acute, subacute or chronic. In the acute phase, MRI is mandatory in order to properly evaluate PCL integrity and associated lesions.

In most cases the causes of the PCL injuries are traffic accidents (45\%), followed in $40 \%$ of instances by contact sports [11]. The mechanism is either by a pretibial blow, hyperextension, or lateral rotation. In a prospective study carried out on 180 patients with PCL reconstructions, $11 \%$ had their ACL reconstructed prior to the PCL. This happened due to either incorrect initial diagnosis or incorrect management [12].

Depending on the acute or chronic setting, the clinical examination may show popliteal hematoma, posterior knee pain or knee instability. One standard exam for a PCL injury is the posterior drawer test.18 (Figure 4). It is essential to refine the difference between this test and the anterior drawer test for ACL injuries, since the posterior drawer may be easily confused with the anterior drawer and may result in a wrong diagnosis and eventually a wrong ligament reconstruction [9]. The fact that ACL injuries are much more common could 
also predispose to such a diagnostic error, with dire consequences.

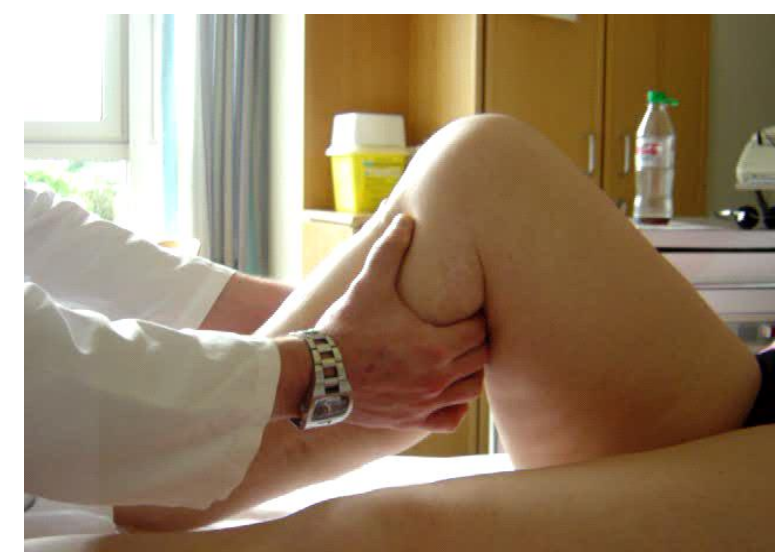

Figure 4 - Posterior drawer test



Figure 5 - PTT measurement in a normal knee

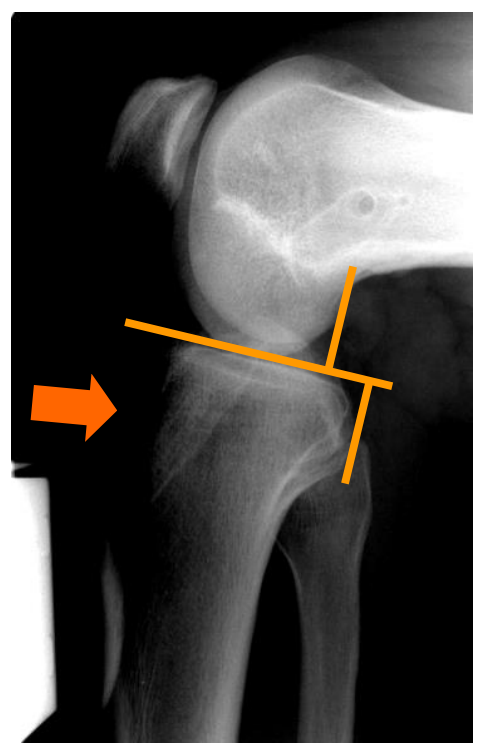

Figure 6 - PTT measurement in a PCL deficient knee
Standard radiographic investigation is essential prior to arthroscopy, to exclude associated fractures or avulsion-fractures. Posterior drawer stress X-rays (Figure 5, Figure 6) compared to the contralateral side are especially relevant for determining the position of the tibia relative to the femur $[19,20]$. When a posterior force is applied to the tibia, the incompetent PCL cannot restrict the posterior tibial translation and the tibia is placed in a posterior position to the femur. In order to evaluate this displacement, the lateral X-ray should be as pure as possible with the medial femoral condyle superimposing the lateral femoral condyle. Even though in practice this is not so common and the rotation cannot be antagonized perfectly every time, a precise measurement is made by drawing a perpendicular line between the intersection of the two femoral condyles and a parallel line tangent to the tibial plateau.

The gold-standard imagistic exam for a PCL injury, prior to arthroscopy, is the MRI, especially in the acute setting. Besides indirect findings such as the floppy-ACL sign (Figure 7) or direct finding of the PCL midsubstance tear or avulsion, it can show other associated lesions such as bone bruises, collateral ligaments or menisci tears [9]. Tewes et al. studied the relevance of MRI examination in chronic cases of PCL injuries and found that even though the ligament might look healed, the functional status of the affected knee would still be altered, due to ligament elongation [21].

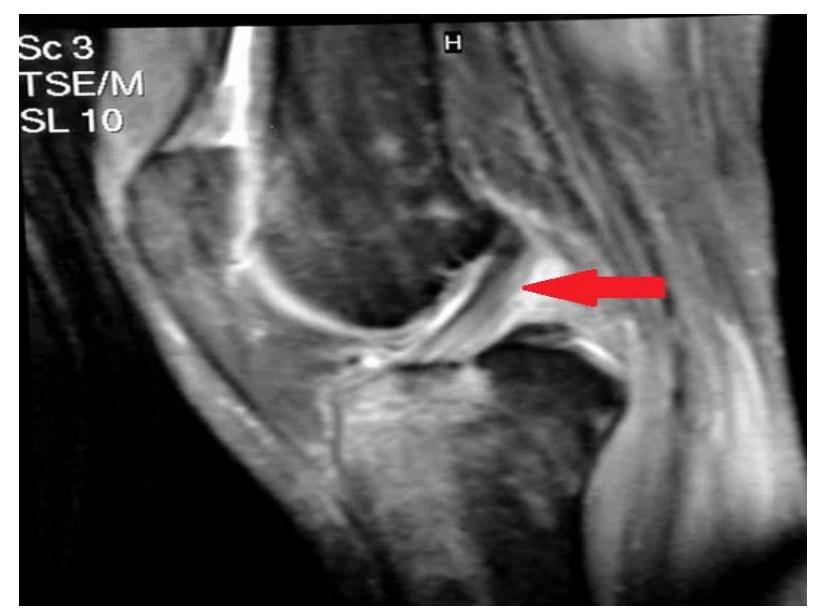

Figure 7 - T2 sagittal view: Floppy-ACL sign PTT is visible too.

When the posterior drawer test is performed intraoperatively in the PCL deficient knee 
(Figure 8), the ACL loses its tension, becomes floppy, revealing the PCL incompetence. Therefore, confusion between ACL and PCL injuries is possible even with arthroscopic examination [9] In comparison with the ACL pseudolaxity shown in arthroscopy prior to the ligament reconstruction, the MRI below (Figure 9) shows a postoperative image of a taut ACL after PCL reconstruction and fixation in maximal anterior drawer.

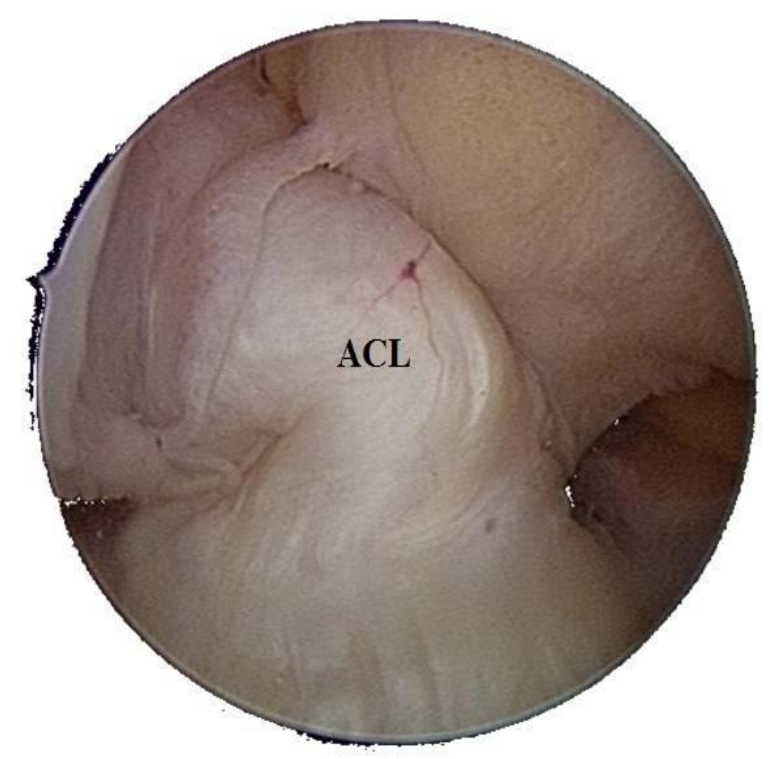

Figure 8 - Floppy-ACL sign in anterior arthroscopic view of the right knee

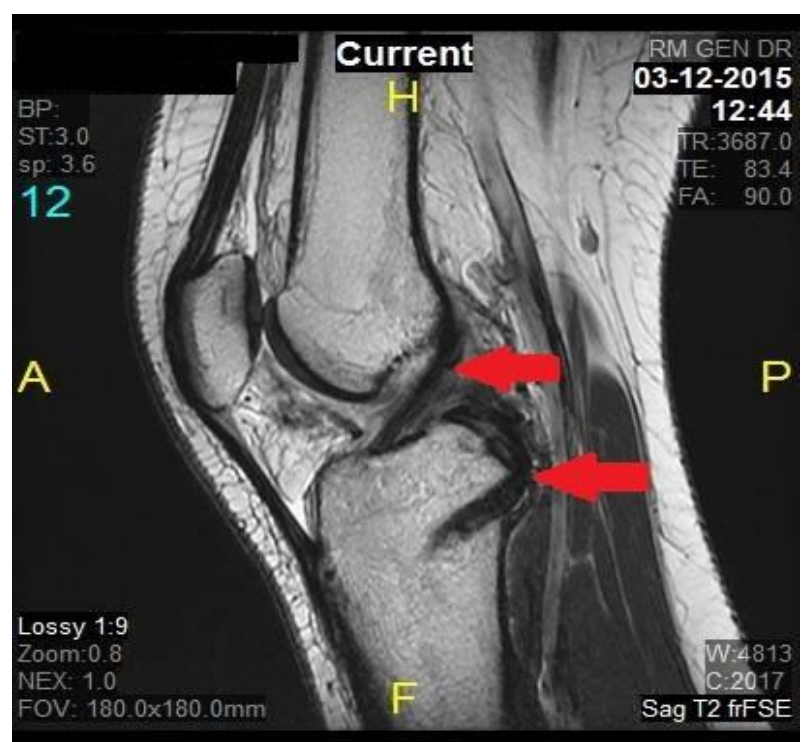

Figure 9 - T1 Sagittal image of the right knee showing the ACL tensioned and the PCL graft

We aimed to review the diagnostic and therapeutic options in such delicate cases of floppy ACL reconstructions, as well as to highlight the importance of correct initial PCL deficiency diagnosis.

Materials and Methods

We retrospectively analyzed the management of a series of five consecutive cases of iatrogenic floppy ACL reconstructions which resulted in fixed posterior drawer. All patients were young athletes with a mean age of 23.6 years. Four of them were males, 3 football players and 1 rugby player and one female, a handball player.

They were referred to our department during their ACL rehabilitation program which was failing. All patients had patellofemoral pain and some degree of meniscal or chondral damage due to the incorrect tibial loading during weight bearing.

\section{Discussions}

In all cases arthroscopic PCL reconstruction (Figure 10, Figure 11, Figure 12, Figure 13) was performed and the acquired injuries after the initial ACL reconstruction were treated.

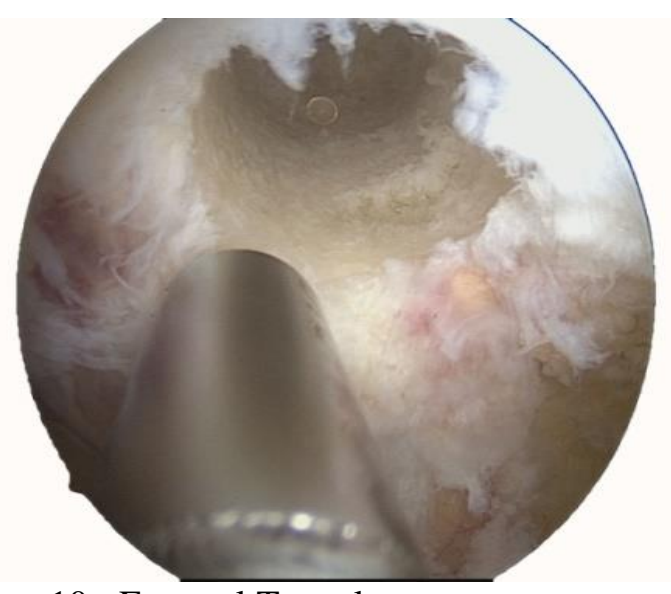

Figure 10 - Femoral Tunnel

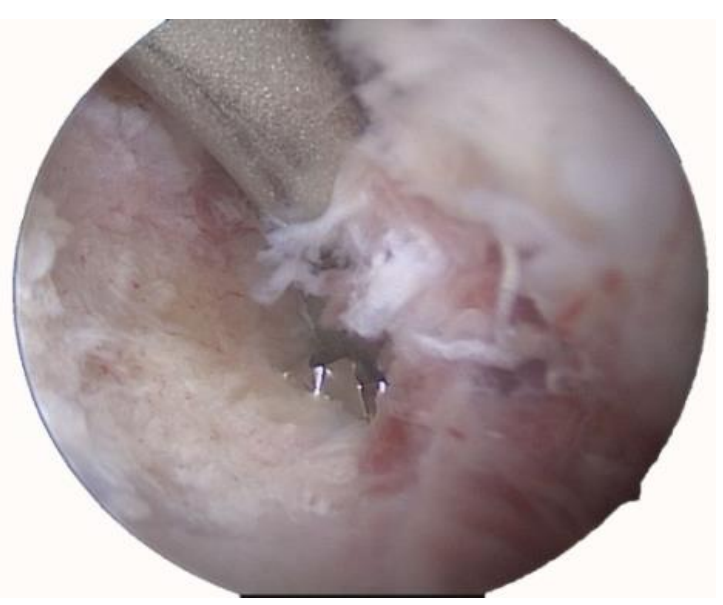

Figure 11 - Postero-medial portal: Tibial tunnel preparation 


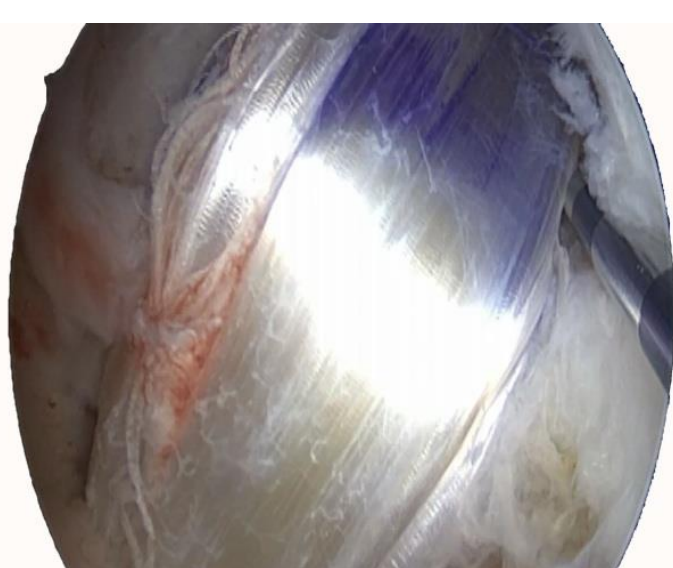

Figure 12 - Anterior view: Graft passage through the femoral tunnel

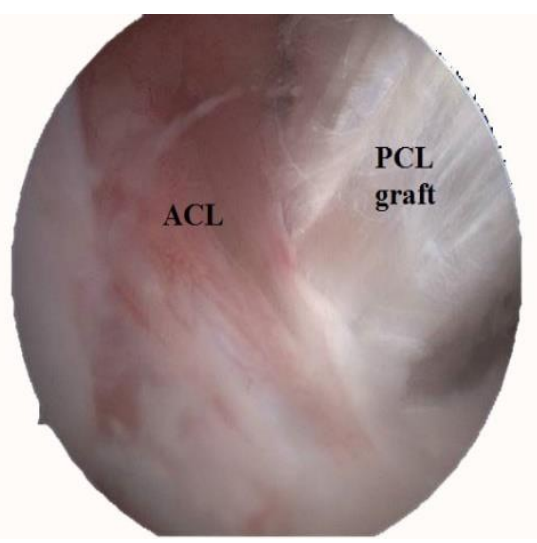

Figure 13 - Anterior view: PCL graft that enhances the tensioning of the ACL

Two cases required techniques to induce fibrocartilage regeneration for the lesions involving the medial femoral condyle, two cases required partial medial meniscectomies and one case required medial meniscus suture. Limited ACL graft release was required in 3 cases to allow for sufficient anterior drawer.

The PCL graft was protected 4 weeks postoperatively with a specific brace (Figure 14) and all patients were enrolled in PCL specific rehabilitation program. All patients regained full range of motion and stable knees.

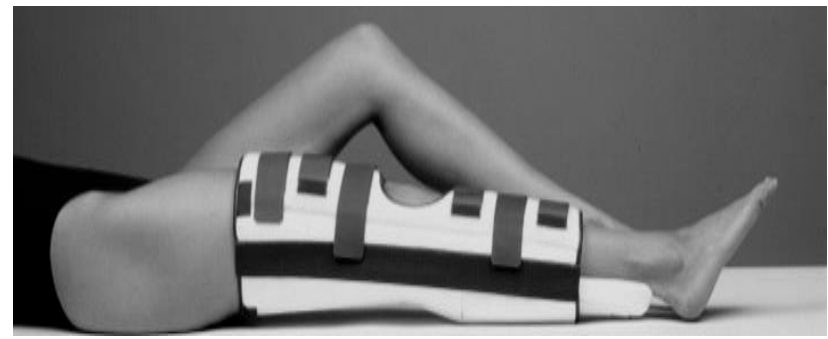

Figure 14 - PCL support brace

\section{Conclusions}

Confusion between PCL and ACL injuries still seems to be high. If not addressed accordingly, the fixed posterior drawer may lead to irreversible damage, especially in the medial compartment and the patellofemoral joint. Even after proper revision surgery, the acquired lesions make the chances of full recovery limited.

We want to put an emphasis on the importance of the right diagnosis at the right time for a PCL injury. If a floppy but intact ACL is reconstructed and the PCL tear is neglected, a secondary, more complex revision surgery is required in order to reestablish the knee's kinematics and stability. In some cases, with fixed posterior drawer, the ACL graft is also compromised. If the ACL tunnels are also incorrect, this unhappy sequence of procedures, of floppy ACL plasty - PCL plasty with ACL release - ACL revision plasty, can be a careerending adventure for an athlete.

\section{References}

[1]Dodds JA, Arnoczky SP: Anatomy of the anterior cruciate ligament: a blueprint for repair and reconstruction.Arthroscopy 10:132, 1994.

[2]Petersen W, Tillmann B: Structure and vascularization of the cruciate ligaments of the human knee joint. Anat Embryol (Berlin) 200:325, 1999.

[3]Kopf S, Musahl V, Tashman S, Szczodry M, Shen W, Fu FH: A systematic review of the femoral origin and tibial insertion morphology of the ACL. Knee Surg Sports Traumatol Arthrosc 17:213, 2009.

[4]Torzilli PA, Greenberg RL, Insall J: An in vivo biomechanical evaluation of anterior-posterior motion of the knee. Roentgenographic measurement technique, stress machine, and stable population. J Bone Joint Surg Am 63:690, 1981.

[5]Wascher DC, Markolf KL, Shapiro MS, Finerman GA: Direct in vitro measurement of forces in the cruciate ligaments. Part I: The effect of multiplane loading in the intact knee. J Bone Joint Surg Am 75:377, 1993.

[6]Amis AA, Gupte CM, Bull AM, Edwards A: Anatomy of the posterior cruciate ligament and the meniscofemoral ligaments, Knee Surg Sports Traumatol Arthrosc 14:257, 2006.

[7]Harner CD, Xerogeanes JW, Livesay GA, Carlin GJ, Smith BA, Kusayama T, Kashiwaguchi S, Woo 
SL: The human posterior cruciate ligament complex: an interdisciplinary study. Ligament morphology and biomechanical evaluation, Am J Sports Med 23:736, 1995.

[8]Pamela K Levangie, Cynthia C. Norkin: Joint Structure And Function A comprehensive Analysis, Fifth Edition, 2011.

[9]Gregory C Fanelli: Posterior Cruciate Ligament Injuries: A Practical Guide to Management. Switzerland: Springer 2015.

[10]Fanelli G.C., Giannotti B.F., Edson C.J : The posterior cruciate ligament arthroscopic evaluation and treatment. Arthroscopy 10:673, 1994.

[11]Schulz MS, Russe K, Weiler A, Eichhorn HJ, Strobel MJ: Epidemiology of posterior cruciate ligament injuries. Arch Orthop Trauma Surg 123:186, 2003.

[12]Weiler A, Jung TM, Lubowicki A, Wagner M, Schöttle PB: Management of posterior cruciate ligament reconstruction after previous isolated anterior cruciate ligament reconstruction.Arthroscopy 23:164, 2007.

[13]Strobel MJ, Weiler A, Schulz MS, Russe K, Eichhorn HJ: Fixed posterior subluxation in posterior cruciate ligament-deficient knees: diagnosis and treatment of a new clinical sign. Am J Sports Med 30:32, 2002.

[14]Ritchie JR, Bergfeld JA, Kambic H, Manning T: Isolated sectioning of the medial and posteromedial capsular ligaments in the posterior cruciate ligament-deficient knee. Influence on posterior tibial translation. Am J Sports Med 26:389, 1998.
[15]Kurosawa H, Yamakoshi K, Yasuda K, Sasaki T: Simultaneous measurement of changes in length of the cruciate ligaments during knee motion. Clin Orthop Relat Res 265:233, 1991.

[16]Dejour H, Walch G, Peyrot J, Eberhard P: The natural history of rupture of the posterior cruciate ligament. Rev Chir Orthop Reparatrice Appar Mot 74:35, 1988.

[17]Boynton MD, Tietjens BR: Long-term followup of the untreated isolated posterior cruciate ligamentdeficient knee. Am J Sports Med 24:306, 1996.

[18]Muller PD Dr Webber: The knee: form, function and ligament reconstruction. Berlin: Springer 1982.

[19]Schulz MS, Russe K, Lampakis G, Strobel MJ. Reliability of stress radiography for evaluation of posterior knee laxity. Am J Sports Med 33:502-6, 2005.

[20]Jackman T, LaPrade RF, Pontinen T, Lender PA. Intraobserver and interobserver reliability of the kneeling technique of stress radiography for the evaluation of posterior knee laxity. Am J Sports Med 36(8):1571-6, 2008.

[21]Tewes DP, Fritts HM, Fields RD, Quick DC, Buss DD. Chronically injured posterior cruciate ligament: magnetic resonance imaging. Clin Orthop Relat Res 335:224-32, 1997.

[22]Grover JS, Bassett LW, Gross ML, et al. Posterior cruciate ligament:MRI imaging. Radiology174:527-30, 1990. 